\title{
Nasogastric Tube Syndrome: Why Is It Important in the Intensive Care Unit?
}

\author{
Taehyun Kim, M.D., Seong Min Kim, M.D., Sung Birm Sohn, M.D., Yeon Ho Lee, M.D., \\ Sang Youn Lim, M.D., and Jae Kyeom Sim, M.D.
}

Department of Internal Medicine, Korea University Guro Hospital, Seoul, Korea

\begin{abstract}
Although the nasogastric tube (NGT) is widely used in critically ill patients, most intensivists do not give much thought to it or its possible complications. NGT syndrome is a rare but fatal complication characterized by throat pain and vocal cord paralysis in the presence of NGT. Recently, we experienced a case of NGT syndrome developed in an 86-year-old female twelve days after NGT insertion. We immediately removed the NGT and secured the airway by tracheostomy. She was treated successfully with an intravenous antibiotic, steroid and proton pump inhibitor and the syndrome did not recur after reinsertion of the NGT.
\end{abstract}

Key Words: nasogastric tube; upper airway obstruction; vocal cord paralysis.

Nasogastric tube (NGT) insertion is a universal procedure in intensive care units (ICUs) for gastric decompression or enteral nutrition (EN). Although indwelling tubes are easy to place and safe, serious complications can occur. NGT syndrome is characterized by throat pain and bilateral vocal cord paralysis, which can cause upper airway obstruction. Despite its lifethreatening potential, NGT syndrome is not well-known. Here we present a case of an elderly woman with NGT syndrome and highlight its significance in ICUs.

\section{Case Report}

An 86-year old woman was admitted to the ICU for pneumonia and septic shock. She had a history of hypertension, diabetes mellitus, and Parkinson's disease. We managed hypoxia with a simple oxygen mask. Due to her altered mental state, oral feeding was not possible and an NGT was inserted. EN was initiated on hospital day 2 but withheld from hospital day 5 through 11 due to cholecystitis. An NGT was kept in place throughout this period. Her clinical course was initially favorable. However, her body temperature and inflammatory markers became elevated again on day 13, and she made a grimace. Two days later, stridor was heard and her blood oxygen saturation began to

Received on May 14, 2015 Revised on June 22, 2015 Accepted on July 9, 2015

Correspondence to: Jae Kyeom Sim, Department of Internal Medicine, Korea University Guro Hospital, 148 Gurodong-ro, Guro-gu, Seoul 08308, Korea Tel: +82-2-2626-2806, Fax: +82-2-2626-1166

E-mail:makyoum2@gmail.com

*No potential conflict of interest relevant to this article was reported. fall. A laryngoscopic examination revealed impaired abduction of the vocal cords, whose internal diameter was reduced to $10 \%$ of the normal. We subsequently performed bronchoscope-guided intubation and percutaneous dilatational tracheostomy. A computed tomography scan of the neck did not show any abnormal findings. We removed the NGT and 
administered intravenous esomeprazole (40 mg) and hydrocortisone $(50 \mathrm{mg})$ twice a day for 5 days. Fifteen days later, vocal cord movement returned. Her mental state was still inadequate for oral feeding and her surrogates refused gastrostomy; thus an NGT was reinserted. Vocal cord paralysis did not recur.

\section{Discussion}

Provision of nutritional support is a crucial component of ICU care and EN is preferred over parenteral nutrition. [1] NGT insertion is simple and minimally invasive and is frequently carried out in critically ill patients. NGT-related complications are not as rare as commonly believed. Most complications are due to mechanical injury of the nose, esophagus, and stomach. Misplacement of the NGT into a pulmonary or intracranial region can also occur.[2,3] NGT syndrome was first named by Sofferman et al.[4] to describe bilateral vocal cord paralysis developed in patients with an NGT. Three pathophysiologic mechanisms were proposed as the cause of NGT syndrome; the dynamic and vertical movements of the larynx against the fixed NGT, compression of the cricoid by the NGT and the cervical spine, and tonic contraction of the cricopharyngeus muscle pulling the NGT against the postcricoid. These induce postcricoid esophageal ulceration and extended inflammation causes esophageal perforation and myositis of the posterior cricoarytenoid (PCA). As a result, vocal cord abduction is compromised.[4] However, esophageal ulceration is not always present. Isozaki et al.[5] reported four patients with a variant of NGT syndrome who did not exhibit pain and did not show esophageal ulceration at autopsy. They hypothesized that the NGT compressed the vasculature of the PCA and led to ischemic damage and muscle paresis. In addition, neurogenic atrophy of the PCA may contribute to the development of NGT syndrome.[6] Sofferman et al.[4] indicated that diabetes was an important risk factor for NGT syndrome and presented the hypothesis that diabetes-associated microvascular disease and immunosuppression made patients vulnerable to NGT syndrome.

Diagnosis of NGT syndrome is challenging. It is uncommon enough to escape being recognized by clinicians and there are no specific signs or symptoms. Throat pain has been thought to be a core symptom of NGT syndrome.[4] However, Brousseau and Kost[7] reviewed 17 cases and found that four patients did not complain of throat pain. Every reported symptom was nonspecific and could be evoked by the NGT itself. Cricoid ulceration, a key process of NGT syndrome, was reported in only seven patients. The review also revealed that onset time varied from 12 hours after tube insertion to 2 weeks after tube removal. Therefore, it is difficult to suspect NGT syndrome based on the time between symptom onset and tube insertion. In our case, the patient had indications typical of NGT syndrome such as diabetes and throat pain. Pain was assumed based on her facial expression. In contrast, she did not show cricoid ulceration. However, it was unclear if cricoid ulceration really did not occur in this case or if it was present but was overlooked.

There is no established treatment for NGT syndrome. Removal of the NGT, tracheostomy, antibiotics, and steroids are the typical options.[7] We removed the NGT and performed tracheostomy. The patient had already received antibiotics, so intravenous steroid and proton pump inhibitor were administered. Tracheostomy is mandatory, because it protects the airway and allows clinicians to evaluate vocal cord function regularly. There is no clear evidence of the benefit of administering antibiotics or steroids but their use is reasonable since they would reduce inflammation and infection. The worsening of inflammation by acid regurgitation aggravated by NGT placement advocates the use of a proton pump inhibitor or an H2-blocker.[8] It takes 2 weeks, on average, to restore normal vocal cord function. Unfortunately, it could take up to 2 months or not happen at all.[7,9] If recovery takes a long time, EN via gastrostomy is worth consideration. We believe that reinsertion of the NGT is an acceptable alternative. Friedman et al.[10] demonstrated that a midline position of the NGT produced more severe inflammation than a lateral position, but that only $6 \%$ of NGTs were placed in this position. Hence, recurrence of NGT syndrome could be greatly reduced or even prevented if its position is checked with neck radiographs.

The pain caused by NGT syndrome is prone to be missed in an ICU where abundant sources of pain exist. On the other hand, patients may not experience or complain about NGT pain due to analgesics, sedatives or underlying diseases. Moreover, upper airway obstruction would be concealed by intubation or tracheostomy. NGT syndrome can cause 
fever or extubation failure and lack of awareness of NGT syndrome could result in unnecessary tests, treatments, and deterioration of the patient's condition. Intensivists should have a suspicion of NGT syndrome in patient with an NGT presenting with unexplained fever, throat pain, or respiratory distress.

\section{ORCID}

Jae Kyeom Sim http://orcid.org/0000-0003-0873-2807

\section{References}

1) McClave SA, Martindale RG, Vanek VW, McCarthy M, Roberts P, Taylor B, et al: Guidelines for the provision and assessment of nutrition support therapy in the adult critically ill patient: Society of Critical Care Medicine (SCCM) and American Society for Parenteral and Enteral Nutrition (A.S.P.E.N.). JPEN J Parenter Enteral Nutr 2009; 33: 277-316.

2) Yardley IE, Donaldson LJ: Patient safety matters: reducing the risks of nasogastric tubes. Clin Med 2010; 10: 228-30.

3) Psarras K, Lalountas MA, Symeonidis NG, Baltatzis M,
Pavlidis ET, Ballas K, et al: Inadvertent insertion of a nasogastric tube into the brain: case report and review of the literature. Clin Imaging 2012; 36: 587-90.

4) Sofferman RA, Haisch CE, Kirchner JA, Hardin NJ: The nasogastric tube syndrome. Laryngoscope 1990; 100: 962-8.

5) Isozaki E, Tobisawa S, Naito R, Mizutani T, Hayashi $\mathrm{H}$ : A variant form of nasogastric tube syndrome. Intern Med 2005; 44: 1286-90.

6) Friedman M, Toriumi DM: Esophageal stethoscope. Another possible cause of vocal cord paralysis. Arch Otolaryngol Head Neck Surg 1989; 115: 95-8.

7) Brousseau VJ, Kost KM: A rare but serious entity: nasogastric tube syndrome. Otolaryngol Head Neck Surg 2006; 135: 677-9.

8) Apostolakis LW, Funk GF, Urdaneta LF, McCulloch TM, Jeyapalan MM: The nasogastric tube syndrome: two case reports and review of the literature. Head Neck 2001; 23: 59-63.

9) Leclerc C, Perhirin M, De Rugy MG, Valdazo A: Severe laryngeal injury due to a nasogastric tube. Ann Fr Anesth Reanim 2002; 21: 306-9.

10) Friedman M, Baim H, Shelton V, Stobnicki M, Chilis T, Ferrara T, et al: Laryngeal injuries secondary to nasogastric tubes. Ann Otol Rhinol Laryngol 1981; 90(5 Pt 1): 469-74. 